\title{
KAWASAKI DISEASE IN GHANA: CASE REPORTS FROM KORLE BU TEACHING HOSPITAL
}

\author{
E. V. BADOE ${ }^{1}$, J. NEEQUAYE ${ }^{1}$, J. O. OLIVER-COMMEY ${ }^{1}$, J. AMOAH ${ }^{2}$, A. OSAFO ${ }^{2}$, I. \\ ARYEE $^{2}$ and M. Y. NYARKO ${ }^{3}$ \\ ${ }^{1}$ Department of Child Health, University of Ghana Medical School, ${ }^{2}$ Department of Child Health, \\ Korle Bu Teaching Hospital, ${ }^{3}$ Princess Marie Louise Hospital, Accra, Ghana
}

Corresponding Author: Dr. Ebenezer V. Badoe

Conflict of interest: None declared

\section{SUMMARY}

Kawasaki disease, an acute febrile vasculitis, predominantly affects children under the age of 5 years and is thought to be a rare disease in the developing world. . It has previously never been reported in Ghana. We report 3 cases from February, 2007 to February, 2008. This potentially serious disease has no definitive diagnostic test and it is not unusual for diagnosis to be delayed with serious consequences. Any child with irritability and persisting fever ( $>5$ days) not responding to antipyretics and antibiotics, accompanied by a rash and non purulent conjunctivitis should be suspected to have Kawasaki disease.

Keywords: Kawasaki Disease, Coronary Arterial Aneurysms, Aspirin, Ghana.

\section{INTRODUCTION}

Kawasaki disease (KD) is a systemic vasculitis affecting small to medium sized arteries but with a predilection for the coronary vessels and most common in Japan where the annual reported incidence is 108 per $100,000 .{ }^{1}$ Dr Tomisaku Kawasaki published a case series of 50 children in 1967. He termed it "acute febrile mucocutaneous syndrome". 2 Children under the age of 5 years are predominantly affected with a peak incidence at 9 to 11 months. ${ }^{3}$ The exact cause has not been established but there is considerable support for it being due to an infectious agent causing disease among genetically vulnerable individuals.

This is probably the result of superantigen activity-potent stimulators of $\mathrm{T}$ cells bearing specific $\mathrm{V}$ beta receptors may play a role in the pathogenesis of coronary arteritis. ${ }^{4}$ Research suggests that the acute vasculitic process is associated with a number of immunoregulatory changes including an oligoclonal immune response where $\operatorname{IgA}$ plasma cells are felt to play a central part. ${ }^{5}$
E-mail: benbadoe@yahoo.co.uk

We report three cases with characteristics that fit the case definition accepted by the American Heart Association Committee on Rheumatic Fever, Endocarditis and Kawasaki Disease. ${ }^{6}$ These include a febrile illness persisting for 5 days or more with any four of the following: changes in extremities (initially erythema and oedema of palms and soles, and peeling of skin from fingertips and toes in the convalescent stage); a diffuse and polymorphic rash (macular, macula-papular, urticarial, or morbilliform) starting in the first few days and lasting a week before fading. There are no vesicles or crusts; oral changes (erythema of lips or oropharynx, strawberry tongue due to prominent papillae or fissuring of the lips); bilateral conjunctival injection (non-suppurative); and non-purulent cervical lymphadenopathy (bilateral or unilateral).

The diagnosis is based on the clinical criteria and the exclusion of other diseases in our environment particularly measles, Group A beta Haemolytic Streptoccocal infection, sickle cell dactylitis, primary cervical adenitis and pyrexia of undetermined origin (PUO). The cases presented met the full criteria for a complete case. There was no other reasonable cause for our findings.

\section{CASE 1}

A previously healthy four year old Ghanaian female presented in February 2007 with a 3-day history of painful neck swelling and high grade fever. The swelling had gradually increased in size and was associated with painful swallowing. She was now anorexic and had vomited once. Her parents denied any history of contact with a chronic cough patient. She was on oral antibiotics and paracetamol but there had been no improvement.

Her past medical history revealed she had been managed for a sub-ovarian mass and pyosalpingitis months earlier. 
Examination revealed an acutely ill girl who was febrile $\left(39^{\circ} \mathrm{C}\right)$, not pale and with a prominent right cervical lymph node measuring $5 \times 5 \mathrm{~cm}$. The lymph node was tender, felt warm to touch, discrete and firm. Cardiovascular examination revealed a soft, systolic murmur as the only abnormality. The throat and ears looked normal. All other systems were normal. A differential diagnosis of lymphoproliferative disease, leukaemia, juvenile rheumatoid arthritis and Kawasaki disease was made.

She was started on intravenous antibiotics (cefuroxime, gentamicin), syrup paracetamol and treated for malaria as well. After 48 hours on admission, her temperature remained high $\left(39.3^{\circ} \mathrm{C}\right)$, and she had now developed bilateral non-purulent conjunctivitis. The lips, tongue and oropharynx were noted to be injected. Her palms and soles also became reddened. By this time a diffuse maculopapular rash was noted on the trunk and upper limbs.

She now complained of painful fingers and these were found to be swollen, tender and erythematous. She was very irritable and refused to walk. Her full blood count showed a leucocytosis (WBC $15 \times 109 / \mathrm{L}$ with $79 \%$ neutrophils), Hb of $10.1 \mathrm{~g} / \mathrm{dL}$ and a platelet count of $313 \times 109 / \mathrm{L}$. No malaria parasites were found and her ESR was markedly raised to $90 \mathrm{~mm} / \mathrm{hr}$. Blood film comment revealed normocytic, normochromic cells with increased WBC, neutrophilia with band forms and coarse toxic granulations.

Platelets were abundant. The C-reactive protein was markedly elevated at $643 \mathrm{mg} / \mathrm{L}$ (normal 0 $10 \mathrm{mg} / \mathrm{L}$ ), Mantoux test was negative and a 'Monospot' screen for infectious mononucleosis was also negative. Urine routine examination and culture was normal. LFTs were normal. An echocardiogram revealed a small pericardial effusion consistent with a myocarditis. Antibiotics were changed to ceftriaxone and flucloxacillin but the temperature continued to swing. . She was started on high dose aspirin $(80 \mathrm{mg} / \mathrm{kg}$ day in divided doses) on day 8 of the illness and all antibiotics stopped. Her lymph node rapidly reduced in size, resulting in a planned biopsy being deferred.

From the second week of admission patient developed desquamation of both axillae and perineum and this was soon followed by peeling of the skin of the digits. Her temperature finally settled after 3 weeks on admission and she was discharged on a low dose of aspirin at $2 \mathrm{mg} / \mathrm{kg} /$ day. Repeat echo- cardiograms at 2 and 6 weeks post discharge were normal. Her aspirin therapy was then stopped at 6 weeks. She remains well.

\section{CASE 2}

A seven month old male infant was referred to the Korle $\mathrm{Bu}$ Teaching Hospital, Accra in November 2007 ,

on suspicion of Kawasaki disease. He had been on admission for 14 days at the Princess Marie Louise Children's Hospital, also in Accra with a 16-day history of persistent pyrexia unresponsive to treatment. He was previously well until he developed a persistent fever which on most days was above $38^{\circ} \mathrm{C}$. He was noted to be very irritable at the time of presentation, although alert.

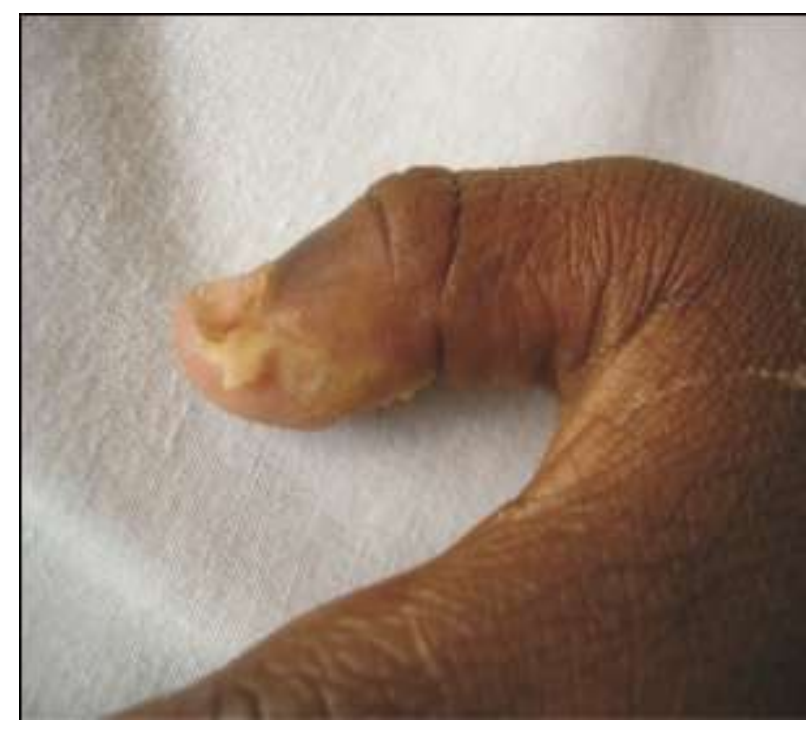

Figure1 Kawasaki Disease - Characteristic fingertip peeling

The course of the present illness had been a nonpurulent conjunctivitis a maculopapular rash, sore mouth and reddened lips on day 2 and cough on day 3 that was present but resolving. The dorsum of both hands became swollen on day 7 followed by swollen proximal and middle phalanges of both hands. Peeling of the skin of these fingers was noted on day 12 and was present at presentation.

He had been given a variety of antibiotics while on admission including 12 days of ceftriaxone, 6 days of cloxacillin, 4 days of cefuroxime, and 3 days of amikacin. On presentation, he was febrile $\left(38^{\circ} \mathrm{C}\right)$ and irritable but alert. He had a dry barking cough and evidence of healed stomatitis. He was neither pale nor icteric. He had bilateral cervical lymphadenopathy. 
The back of both hands were swollen, as well as the proximal and middle phalanges. The central nervous system, respiratory, cardiovascular, gastro-intestinal, throat and ear examinations were normal. Differential diagnoses entertained were Kawasaki disease, sepsis, tuberculosis, infective endocarditis and immunosuppression.

Investigations done revealed the following: $\mathrm{Hb}-$ $7.2 \mathrm{~g} / \mathrm{dL}, \quad \mathrm{WBC}-13.5 \times 109 / \mathrm{L}, \quad$ platelets $571.4 \times 109 / \mathrm{L}$. Blood film comment was normal. ESR - initially $80 \mathrm{~mm} / \mathrm{hr}$, and then at week 3, $64 \mathrm{~mm} / \mathrm{hr}$. Hb genotype - AA. Blood culture - no bacterial growth. ASO titre - non reactive. Retroviral antibody test - non reactive. Mantoux test non reactive. Urine routine exam and culture/sensitivity was normal. Chest X-ray and electrocardiogram were normal.

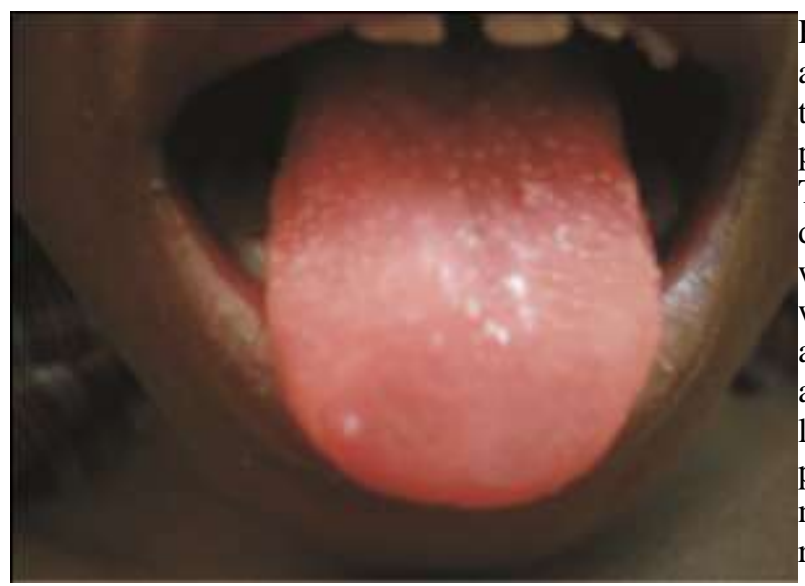

Figure 2 Kawasaki Disease - mucositis with "strawberry tongue"

He was treated as a case of Kawasaki disease and put on high dose aspirin, $100 \mathrm{mg} / \mathrm{kg}$ in divided doses daily followed by low dose aspirin $5 \mathrm{mg} / \mathrm{kg}$ daily. The temperature settled within 3 days of starting high dose aspirin and was switched to low dose aspirin. He was discharged home on day 3 of starting low dose aspirin. He was followed up over 2 months and remained well. Repeat echocardiogram was normal.

\section{CASE 3}

A five year old Ghanaian male presented in January 2008 with a 5 day history of fever, red eyes and sore throat incompletely relieved by paracetamol, artesunate/amodiaquine, and amoxilclavunate given to him by his mother. History revealed that a diffuse maculopapular rash developed 6 days after the onset of the fever that spread from the face, to the neck and rest of the body. He developed vomiting and diarrhoea a day before presentation. He complained of pain on swallowing. This progressed to abdominal pain and arthralgia. The referring hospital had started intravenous ciprofloxacin and intravenous hydrocortisone.

The vital signs on presentation were a temperature of $39.5^{\circ} \mathrm{C}$, heart rate of 120 beats per minute and a respiratory rate of 32 cycles per minute. Blood pressure was 100/60 mmHg. A soft, non-radiating systolic murmur was heard at the apex, and the liver was found to be tender and enlarged $(2-3 \mathrm{~cm}$ below the costal margin). He was maintained on intravenous ciprofloxacin and blood investigations ordered. On the second day of admission, he developed severe abdominal pain and passed loose stools overnight.

He was noted to be very irritable, febrile $\left(39^{\circ} \mathrm{C}\right)$ and was guarding in the right iliac fossa. His tongue now appeared very red with prominence of papillae. There was no cervical lymphadenopathy. The rash was more intense, with large areas of desquamation on the trunk. Throat examination was, however, normal. By this time, neck stiffness was also noted, and he developed swollen hands and feet. Tenderness in the right hypochondrium and peri-umbilical region was still present with the liver size now documented as $4 \mathrm{~cm}$. A lumbar puncture was done with normal results and a diagnosis of Kawasaki Disease was entertained. Aspirin in high dose $100 \mathrm{mg} / \mathrm{kg}$ was started in 4 divided daily doses.

The following day, he was noted to be still irritable, looked ill and febrile $\left(38.5^{\circ} \mathrm{C}\right)$. He developed cracked dry lips, with desquamation of skin around the neck and chest. Both hands and feet remained swollen. Liver size was now $6 \mathrm{~cm}$ and very tender. A surgical opinion was sought, and a mucocoele was diagnosed. The results of blood tests were as follows. $\mathrm{Hb}-9.0 \mathrm{~g} / \mathrm{L}$, WBC $-17.6 \times 109 / \mathrm{L}$, platelets - 312x109/L. ESR was $110 \mathrm{~mm} / \mathrm{hr}$. Malaria parasites and sickling screen were negative. Blood cultures were negative for bacterial organisms. Liver function tests showed increased serum direct bilirubin and decreased albumin $(32 \mathrm{~g} / \mathrm{L})$ levels.

Chest X-ray showed cardiomegaly with a cardiothoracic ratio of 0.62 . Echocardiography showed normal chambers and trivial mitral regurgitation. No evidence of aortic aneurysm was seen. Abdominal ultrasound illustrated a gall bladder grossly dilated measuring 10.52 x 4.79 x $4.28 \mathrm{~cm}$ with a volume of $112.9 \mathrm{~cm}^{3}$. 
Subsequent liver function tests were deranged. CT scan of gall bladder showed gross dilation. His temperature settled after 4 days when high dose aspirin was started at $100 \mathrm{mg} / \mathrm{kg}$ and all antibiotics stopped. Low dose aspirin was started and he was discharged after 13 days on admission. He completed 4 weeks of low dose aspirin. His repeat echocardiogram was normal. His last review in late March 2008 was entirely normal.

\section{DISCUSSION}

All three children showed signs of complete Kawasaki disease and recovered by the third week. The diagnosis was based on well established clinical criteria as stated earlier. The ESR was raised in all patients and they all had a neutrophil leucocytosis. Case 2 had a high platelet count, which is usually raised towards the end of the second week. None had echocardiographic evidence of aneurysm formation.

Cases 1 and 2 had typical lymphadenopathy and all three cases had a rash and changes of the extremities (Figure1 and 2). Cervical lymphadenopathy is found in $50 \%$ of cases. Most often there is a painful and solitary enlarged lymph gland $>1.5$ $\mathrm{cm}$ in diameter which is non-purulent. ${ }^{7}$ Case 3 showed other recognized features of KD such as a heart murmur, cardiomegaly, vomiting, abdominal pain and hydrops of the gall bladder.

A summary of the non-principal signs of KD include cardiovascular (heart murmur, gallop rythym, cardiomegaly, pericardial effusion.) gastrointestinal tract (diarrhoea, vomiting, abdominal pain, hydrops of gall bladder, mild jaundice), blood (leucocytosis with shift to the left, thrombocytosis, increased ESR and CRP, hypoproteinaemia). urine (proteinuria, leucocytes in urine), skin (redness at site of BCG scar), respiratory(cough) Joint (pain, swelling) and neurological (CSF pleocytosis). ${ }^{8}$ Pyrexia was unremitting in all three cases and responded promptly to aspirin therapy. All the children were typically miserable.

Irritability is an important sign, which was present in all three children, although not included as one of the diagnostic criteria. The exact mechanism of the irritability is unclear, but it may be related to the presence of aseptic meningitis. ${ }^{9} \quad$ The commonest potentially life threatening complication of KD include coronary artery dilation and aneurysm formation, occurring in $10-15 \%$ of patients during the acute stage usually 6-8 weeks from the onset of the illness. ${ }^{10}$
Useful investigations in KD include chest $\mathrm{x}$-ray, electrocardiogram, echocardiogram, renal and liver function tests, dip test for urine protein, C-reactive protein, ESR and blood cultures. Incomplete KD should be considered in all children with unexplained fever for more than 5 days associated with 2 or 3 of the principal clinical features outlined above. It is more common in young infants, making accurate diagnosis and timely treatment especially important in these young patients who are at substantial risk of developing coronary abnormalities. ${ }^{11}$ Standard treatment for KD involves a combination of intravenous immunoglobulin (IVIG) and aspirin. $^{12}$ Intravenous Immunoglobulin was not available to treat our patients.

Aspirin has been considered beneficial in controlling pyrexia, conjunctivitis and mucositis. ${ }^{13}$ In the acute phase it is given in anti-inflammatory doses of $80-100 \mathrm{mg} / \mathrm{kg} /$ day until a febrile for $2-3$ days. Following defervescence aspirin is given as an anti-platelet agent in a dose of $2-5 \mathrm{mg} / \mathrm{kg}$ once daily, the duration dependent on findings at echocardiography. Recent reviews recommend the higher dose of $100 \mathrm{mg} / \mathrm{kg}$ in the acute phase of the illness. $^{14}$

\section{CONCLUSION}

Kawasaki disease should be considered as a possibility in any child with a prolonged fever (lasting more than 5 days) and a rash. Other diagnostic features should then be looked for.

\section{REFERENCES}

1. Yanagawa H, Nakaruma Y, Yashiro M, et al. Incidence survey of Kawasaki disease in 1997 and 1998 in Japan. Pediatrics 2001; 107:E33

2. Kawasaki T. Acute febrile mucocutaneous syndrome with lymphoid involvement and specific desquamation of the toes and fingers in children. Jpn J Allergy 1967;16:178-222

3. Royle JA, Williams K, Elliot E, et al. Kawasaki disease in Australia,1993-95 Arch Dis Child 1998;78:33-9

4. Brogan PA, Shah V, Baggar A, et al. T cell V beta receptors in childhood vasculitidies. Clin Exp Immunol 2003; 31, 517 - 527.

5. Rowley AH, Eckerley CA, Jack HM, et al. 1g A plasma cells in vascular tissue of patients with Kawasaki syndrome. J Immunol 1997;159: 5946 - 5955

6. American Heart Association Committee on Rheumatic fever, Endocarditis, and Kawasaki disease. Diagnostic guidelines for Kawasaki disease. Am J Dis Child 1990;144:1218-9 
7. Maconochie IK. Kawasaki disease. Arch Dis Child Educ Pract Ed 2004;89:ep3-8

8. Newburger JW, Takaahashi M, Geerber MA et al. Diagnosis, Treatment, and Long Term management of Kawasaki Disease. Circulation 2004;110:2747-2771

9. Brogan PA, Bose A, Burgner D, Shingadia D, Tulloh R et al. Kawasaki disease: an evidence based approach to diagnosis, treatment and proposals for future research. Arch Dis child 2002:86:286-290

10. Fujita Y, Nakamura Y. Sakata K et al. Kawasaki disease in families. Pediatrics 1989: 84: 666-669
11. Burns JC, Wiggins JW Jr., Toews WH et al. Clinical spectrum of Kawasaki disease in infants younger than 6 months of age. J Pediatr.1986;109:759-763

12. Brogan PA, Bose A, Burgner D.et al. Kawasaki Disease: an evidence based approach to diagnosis, treatment, and proposals for future research. Arch Dis Child 2003 Jan;88(1):91.

13. Umuzewa T, Matsuo N, Saji T. Treatment of Kawasaki disease using the intravenous aspirin anti-inflammatory effect. Acta Paediatr Jpn 1992;34:548-58

14. Shulman ST. High dose aspirin in Kawasaki disease. J Pediatr 199;134:523-4 